International Braz J Urol

Vol. 34 (4): 492-502, July - August, 2008

\title{
In Vitro Evidence for a New Therapeutic Approach in Renal Cell Carcinoma
}

\author{
Carmine Pittoggi, Gianni Martis, Giorgia Mastrangeli, Bruno Mastrangeli, Corrado \\ Spadafora
}

SBGSA (CP, CS), Italian National Institute of Health, Rome, Italy, Department of Urology (GM, BM), San Camillo De Lellis Hospital, Rieti, Italy and MIPI (GM), Italian National Institute of Health, Rome, Italy

\begin{abstract}
Purpose: Renal cell carcinoma (RCC) is the most lethal among the common urologic malignancies, comprising $3 \%$ of all human neoplasias; approximately $40 \%$ of patients eventually die of cancer progression. One third of patients who present with metastatic disease and up to $40 \%$ treated for localized disease generally experience recurrence. RCCs are characterized by high resistance to chemo-, radio- and immunotherapy. We recently discovered an endogenous enzymatic activity, which is particularly expressed in tumorigenic cell, endogenous non-telomerase reverse transcriptase (RT) of retrotrasposon / retroviral origin, as a specific target to induce proliferation arrest in a number of human carcinogenesis in vitro culture cell lines.

Methods: To address this possibility, we have employed RCC primary cell culture testing pharmacological inhibition, in vitro, by two characterized non nucleosidic RT inhibitors, nevirapine and efavirenz; next, we assessed morphological effects and analyzed putative modulation on gene expression profile.

Results: Both treatments reduced cell proliferation rate and induced morphological differentiation and gene expression reprogramming in different RCC analyzed tumor biomarkers.

Conclusion: In this study we describe a new potential therapeutic approach to obtain considerable future benefits in renal carcinoma cure and attempt to establish a new possible pharmacological therapy based on oral drugs administration in renal RCC treatment.
\end{abstract}

Key words: renal cell carcinoma; reverse transcriptase; gene expression; therapy

Int Braz J Urol. 2008; 34: 492-502

\section{INTRODUCTION}

Renal cell carcinomas (RCCs) are the most frequent tumors of the kidney. They comprise 3\% of all human neoplastic diseases and are increasing in incidence. The etiology of RCC remains unknown and although the majority of renal tumors develop as sporadic forms, rare familiar forms and distinct genetic abnormalities have been observed. In particular, it seems that clear cell carcinoma originates from the proximal tubular epithelium in the renal cortex. Molecular analysis of this solid tumor is furthermore complicated by the mixture of tumor cells and normal cells, composed of leukocytes and connective tissue cells. To overcome this problem the tumor cells may be adapted to grow in vitro, as primary cell cultures, in order to obtain a more homogenous cellular material to study the biochemical and molecular changes associated with neoplastic status. 
RCCs can be classified into clear cell, papillary and chromophobe carcinomas based on their histological appearance.

In these tumors, a crucial role for prevention should include accurate prognosis and systematic investigation of gene expression and/or proteomic profile to identify protein changes caused by disease processes (1). The most frequent type of renal cancer is the clear cell variant, accounting for $80-85 \%$ of adult renal neoplasms.

$\mathrm{RCCs}$ are characterized by high resistance to chemo-, radio- and immunotherapy. Recently, new approaches, based on anti-angiogenic drugs, have emerged as potential therapies in the treatment of metastatic renal cell carcinoma (mRCC). Tyrosine kinase receptors (RTKs) are transmembrane proteins involved in signal transduction. Overexpression and/or the structural alteration of different RTKs classes are generally associated with cancer and their abnormal activation often generates cancer growth, angiogenesis and metastatization. Sunitinib malate is a molecule able to link intracellular tyrosine kinase domain of RTKs, which have an anticancer and antiangiogenetic activity. Sunitinib targets selectively vascular endothelial growth factor, KIT, Flt3 and platelet-derived growth factor receptors and the receptor encoded by the ret proto-oncogene. This drug is currently used in the treatment of gastrointestinal stromal tumors resistant to imatinib and mRCC (2). Sorafenib (Nexavar, BAY43-9006) is a new small-molecule, oral, multi-kinase inhibitor for the treatment of patients with advanced RCC. The response rate to sorafenib is partial $(10 \%)$. In sorafenib treated patients there was a significant prolongation of progression-free survival. Overall survival results are still preliminary. The principal toxicities in the sorafenib patients included reversible skin rashes in $40 \%$ and hand-foot skin reaction in $30 \%$ (3). Another compound recently used in mRCC cancer therapy is temsirolimus, a specific inhibitor for the mammalian target of rapamycin kinase. Interferon alpha is widely used for $\mathrm{mRCC}$ but has limited efficacy and tolerability. As compared with interferon alpha, temsirolimus has been shown to improve overall survival among patients with metastatic renal-cell carcinoma and a poor prognosis (4).
This prompted us to evaluate the possibility of applying our findings for a therapeutic application in combination with kinase inhibitors recently used in $\mathrm{mRCC}$, as a new class of biological agents, which have begun to break the resistance barrier (5).

Cancer progression and development have been accompanied by profound changes at cellular and molecular level, involving RNA, DNA and protein function and structure.

Recently, we have defined an endogenous activity, reverse transcriptase (RT), which is particularly expressed in tumor tissues as compared to normal tissues. In vitro cell culture treatment with specific inhibitors of the endogenous RT activity (6) has led to cell cycle arrest and induced morphological differentiation and gene expression reprogramming.

In this study, we used a human renal carcinoma derived primary cell culture to assess the cell cycle progression in vitro demonstrating that RT non nucleosidic inhibitors (nevirapine and efavirenz) induce cell cycle arrest in RCCs, morphological changes and gene markers modulation.

Our results demonstrate the treatment efficacy of these new drugs as potential therapy for in vitro RCC culture.

\section{MATERIALS AND METHODS}

\section{Tissue Samples}

Samples from 3 patients with clear RCC, containing control non tumoral ("normal") and tumor tissue were obtained from fresh nephrectomy specimens. After surgical resection the samples were immediately snap frozen in liquid nitrogen and stored at $-80^{\circ} \mathrm{C}$ at the Department of Urology of the San Camillo De Lellis Hospital in Rieti, Italy. Paraffin sections from each specimen were reviewed by a pathologist and classified histologically according to UICC-TNM.

\section{Primary Tissue RCC Cultures and Derived Cell Line}

Autologous tumor and cortex renal tissue specimens were collected, after surgery, in cold DMEM medium containing $1 \%$ penicillin/streptomycin, $1 \%$ amphotericin, $0.5 \%$ glutamine, $20 \%$ fetal 
calf serum (FCS) and kept at $4^{\circ} \mathrm{C}$ until processing (within $18 \mathrm{~h}$ ). Tissues, normal and neoplastic, were vigorously washed 4-5 times vortexing in phosphatebuffered saline (PBS) $\mathrm{pH} 7.2$ at $37^{\circ} \mathrm{C}$ and minced in $1-\mathrm{mm}^{3}$ fragments, in a Petri dish containing PBS, and vigorously re-washed vortexing with $\mathrm{PBS}$ at $37^{\circ} \mathrm{C}$. The small fragments were left for $1 \mathrm{~h}$ in a dish in the presence of medium at $37^{\circ} \mathrm{C}$ and then 10 pieces were definitively plated in a new $10 \mathrm{~cm}$ Petri dish and covered by DMEM. Four Petri dishes were routinely prepared for each autologous tissue and incubated at $37^{\circ} \mathrm{C}$ in $5 \% \mathrm{CO}_{2}$. The first medium change was performed after 5 days, when the tissue fragments were removed. Cultures were fed twice weekly and passed in new dishes when $90 \%$ confluent after trypsinization and 1:2 split. Aliquots of cells were cryopreserved in $90 \% \mathrm{FCS} / 10 \%$ dimethyl sulfoxide and stored in liquid nitrogen after 1-2 passages. All experiments were conducted on the third passage. Primary RCC cell line obtained was well identified as T1 N0 M0 stage, $\mathrm{II}^{\circ}$ grade, according to standard nomenclature defined by Fuhrman.

\section{Cell Cultures}

Human RCC primary culture cells were seeded in six -well plates at a density of $10^{4}$ to $5 \mathrm{x}$ $10^{4}$ cells/well and cultured in RPMI 1640 medium with $10 \%$ fetal bovine serum. Nevirapine and efevirenz were purified from commercially available Viramune ${ }^{\circledR}$ (Boehringer-Ingelheim) and Sustiva ${ }^{\circledR}$ (Bristol-Myers Squibb) as described (7). The drugs were made 350 and $15 \mu \mathrm{M}$ (final concentration) in dimethyl sulfoxide (DMSO, Sigma Aldrich), respectively, and added to cells $5 \mathrm{~h}$ after seeding, the same DMSO volume ( $0.2 \%$ final concentration) was added to controls. Every $48 \mathrm{~h}$ fresh RT inhibitors-containing medium was changed. Cells were harvested every 96 $\mathrm{h}$, counted in a Burker's chamber and replated at the same density.

\section{RNA Extraction and Semi-quantitative RT- PCR}

Total RNA was extracted from RCC primary cell culture $\left(10 \times 10^{6}\right.$ cell $)$ treated with nevirapine and efavirenz and untreated (CTR); the RNAeasy mini kit (Qiagen, Germany) was used. Trace amounts of contaminant chromosomal DNA was eliminated by incubation with Rnase-free Dnase I (Invitrogen, Carlsbad, CA ), $1 \mathrm{U} / \mu \mathrm{g}$ of total RNA, for $15 \mathrm{~min}$ at room temperature; $200 \mathrm{ng}$ of each RNA sample was used in a oligo(dT) cDNA synthesis, performed using the Thermoscript RT-PCR system (Invitrogen, USA). $2 \mu \mathrm{L}$ for each cDNA sample were PCR amplified using the Platinum Taq DNA Polymerase I (Invitrogen, USA), in a $50 \mu \mathrm{L}$ reaction mixture containing 30 pmoL of specific oligonucleotides (MWGBiotech, Ebersberg, Germany) in an initial 2-min step at $94^{\circ} \mathrm{C}$, followed by cycles of $30 \mathrm{~s}$ at $94^{\circ} \mathrm{C}, 30 \mathrm{~s}$ at $58-62^{\circ} \mathrm{C}$, $1 \mathrm{~min}$ at $72^{\circ} \mathrm{C}$. Each oligo pair was used in sequential amplification series with increasing numbers (30-40) of cycles. PCR products were fractioned through $1.4 \%$ agarose gels and visualized with UV transilluminator light.

Set of primers used for standard PCR are designed forward (fwd) and reverse (rev). Oligonucleotide sequences and expected product sizes are listed below:

NNMT (NM 006169); PCR product size:188 bp

NNMT fwd 5'-tcaagcaggtgctgaagtgt-3'

NNMT rev 5'-atccatgatcaccaggaagc-3'

NNMT int 5' -agcacactgtgtctggatgc-3'

AFP (NM 001134); PCR product size: 113 bp

AFP fwd 5'-agcttggtggtggatgaaac-3'

AFP rev 5'-tcttgcttcatcgtttgcag-3'

AFP int 5' -tccetcctgcattctctgat-3'

CD70 (EF 064709); PCR product size: 110 bp

CD70 fwd 5'-aatcacacaggacctcagcaggacc-3'

CD70 rev 5' -agcagatggecagcgtcacc-3'

CD70 int 5'-agccgtagtaatggaatccca-3'

FRA2 (X 16706); PCR product size: $125 \mathrm{bp}$

FRA2 fwd 5'-ccetgcacacccccatcgtg-3'

FRA2 rev 5'-tgattggtccccgetgctactgctt-3'

FRA2 int 5'-tccttagataatgcatccagtaa-3'

p 27 KIP1 (NM 004064); PCR product size: 184 bp

p 27 fwd 5'-gccetccccagtctctctta-3'

p 27 rev 5'-acagcccgaagtgaaaagaa-3'

p 27 int 5'-caggtagtttggggcaaaaa-3' 
GAPDH (NM 002046); PCR product size: 650 bp Gapdh fwd 5'-ATTCAACGGCACAGTCAAGG-3' Gapdh rev 5'-AAGGTGGAAGAGTGGGAGTT-3' Gapdh int 5'-GGGAAGCCCATCACCATCT-3'.

Internal oligonucleotides were used as probe to hybridize PCR DNA transferred to Hybond N+ nylon filters. After washing, filters were exposed to FUJI XR-E 30 films for the requested time.

\section{Tumor Markers Gene Expression Profile}

The induction of morphological differentiation suggests that critical regulatory genes are modulated in response to the RT inhibitory treatment. This was investigated in semi-quantitative RT-PCR analysis of cultures treated with DMSO alone, or nevirapine or efavirenz for three cycles. In RCC derived primary cell culture, we focused on a set of five genes: the CD70 gene, a new diagnostic biomarker, known as a member of the tumor necrosis factor (ligand) superfamily (8); the FRA 2 gene, Fos Related Antigen 2 , associated also with apoptosis and regulator of cell proliferation, differentiation and transformation (9); the NNMT gene; the AFP gene; p27KIP1 gene.

RT-PCR results, in Figure-1, indicate that the CD70 gene and the FRA 2 gene were markedly down - regulated, in contrast with not treated tumor cells where expression levels for the same genes were considerably higher; whereas, the AFP gene, which encodes for a major plasma protein, which expression in adults is often associated with hepatoma and teratoma (10), and is thought to be the fetal counterpart of serum albumin, in RT-inhibited RCC, results in a down regulation of its mRNA expression level. In contrast, the NNMT gene, encoding for a cytokine that belongs to the tumor necrosis factor ligand family, involved in $\mathrm{T}$ cell antigen-presenting cell interactions, and along with CD70 shown to provide CD28-independent costimulatory signals (11), results in mRNA expression levels similar to DMSO control expression. We extended mRNA expression to another possible tumor marker: p27 KIP1. This gene encodes a cyclin-dependent kinase inhibitor, which shares a limited similarity with CDK inhibitor CDKN1A/p21. The encoded protein binds to and prevents the activation of cyclin E-CDK2 or cyclin D-CDK4 complexes and thus control the cell cycle progression at G1. The degradation of this protein is required for the cellular transition from quiescence to the proliferation state. Our results show a significant p27 over-expression in nevirapine and efavirenz treated cells, confirming that the p27 expression increase was correlated to a decreasing tumor stage (12).

Thus, RT inhibitory drugs modulate the expression of critical genes implicated in the development of transformed cells, concomitantly with the induction of differentiation-like state relative to quiescence; also, this reprogramming is reversible and is abolished when RT-inhibition is released (data not shown).

\section{Preparation of Cell-Free RCC Lysates and RT Activity Assay}

Cell free RCC lysates were prepared by lysing control, nevirapine and efavirenz treated

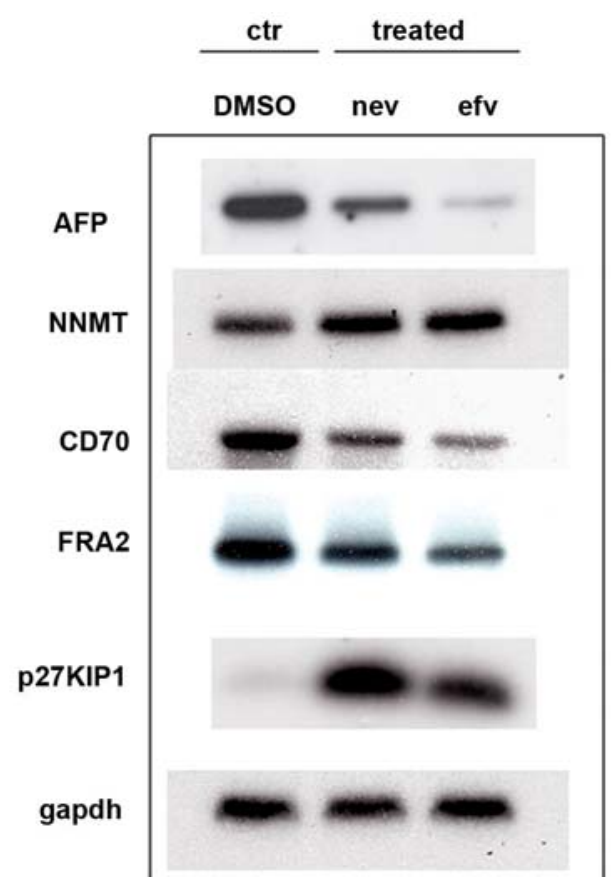

Figure $1-R T$ inhibitors modulate gene expression in primary RCC cell culture. RNA extracted from cells treated with DMSO (ctr), nevirapine (nev) or efavirenz (efv), was amplified by RT$P C R$, blotted and hybridized with internal oligonucleotides. 
RCC cells grown till the third cycle $(288 \mathrm{~h})$ in vitro, using ice-cold lysis buffer $(10 \mathrm{mM}$ Tris$\mathrm{Hcl}, \mathrm{pH}=7.5 ; 1 \mathrm{mM} \mathrm{MgCl}$; $1 \mathrm{mM}$ EGTA; $0.1 \mathrm{mM}$ PMSF; 5 mM $\beta$-mercaptoethanol; 0.5\% CHAPS; $10 \%$ glycerol).

Cell lysates $\left(5 \times 10^{6}\right.$ cells $/ 100 \mu \mathrm{L}$ of lysis buffer) were subjected to three freeze-and-thaw (liquid nitrogen $/ 37^{\circ} \mathrm{C}$ ) cycles, incubated for $30 \mathrm{~min}$ on ice and centrifuged for $30 \mathrm{~min}$ at $14,000 \mathrm{rpm}$ at $4^{\circ} \mathrm{C}$. The supernatant containing the RT activity was aliquoted, quickly frozen in dry ice and stored at $-70^{\circ} \mathrm{C}$.

The protein concentration was determined by the standard Coomassie (Pierce Chemical Co., Chester, England). RT was tested in a PCR-based (PBRT) assay as previously described (3) with minor modifications. Briefly, PBRT reactions contained cells lysate aliquots corresponding to 6 ug of protein, $10 \mathrm{ng}$ of bacteriophage MS2 RNA (Roche Diagnostics, Hertfordshire, England), 50 $\mathrm{mM}$ Tris acetate $(\mathrm{pH} 8.4), 75 \mathrm{mM} \mathrm{K}$ acetate, 40 $\mathrm{mM} \mathrm{Mg}$ acetate, $5 \mathrm{mM}$ DTT, $1 \mathrm{mM}$ of four nucleotide triphosphate mix, $2 \mathrm{U}$ of Rnase OUT and 30 pmoL of MS2 reverse primer (R) (see below for the sequence) in a final volume of $20 \mathrm{uL}$. Reaction mixture were incubated at $55^{\circ} \mathrm{C}$ for 1 hour followed by $5 \mathrm{~min}$ at $85^{\circ} \mathrm{C}$. One microliter of $\mathrm{E}$. Coli RNaseH (2U/uL) was added to each sample and further incubated at $37^{\circ} \mathrm{C}$ for $20 \mathrm{~min}$. Control reactions were set up by omitting cell lysate (negative controls), or adding $1 \mathrm{uL}$ of ThermoScript RT (Invitrogen, Karlsruhe, Germany) $15 \mathrm{U} / \mathrm{uL}$ (positive control). Two microliters from each reaction were mixed with 30 pmoL each of forward (F) $5^{\prime}$ TCCTGCTCAACTTCCTGTCGAG-3' and reverse (R) 5'-CATAGGTCAAACCTCCTAGGAATG-3' MS2 primers and PCR-amplified using ThermoScript RT PCR kit (Invitrogen). PCR conditions were as follows: $95^{\circ} \mathrm{C}$ for $2 \mathrm{~min}$; followed by 30 cycles of $94^{\circ} \mathrm{C}$ for $30 \mathrm{sec}, 58^{\circ} \mathrm{C}$ for $45 \mathrm{sec}$ and $72^{\circ} \mathrm{C}$ for $1 \mathrm{~min}$. The amplification product is a 112-bp DNA fragment spanning positions 21-132 at the 5 ' end of the MS2 RNA (GeneBank J02467). PCR products were fractioned through $1.5 \%$ agarose gel electrophoresis; Southern blotted filters were hybridized with end-labeled internal oligonucleotide, 5'-TTAATGTCTTAGCGAGACGC-3'.

\section{RESULTS}

\section{In Vitro Treatment with RT Inhibitors Induces RCCs Proliferation Arrest}

The in vitro treatment with RT non-nucleosidic inhibitors induced cell growth arrest in several human cell line cultures (6) and caused, in vivo, a growth arrest in nude mice inoculated human tumor (13). In a previous work, we reported that the RT inhibitor nevirapine, largely used in anti-HIV therapy, blocks the enzymatic activity of endogenous RTs in non infected proliferating cells, as revealed using a highly sensitive RT-PCR based in vitro assay (7), and, concomitantly, reduces the growth of human primary RCC cell culture (T1 N0 M0 stage, II $^{\circ}$ grade) to prolonged exposure to RT inhibitors. Two well-defined RT inhibitors, nevirapine and efavirenz, were used. Primary culture cells were passed, counted and replated every $96 \mathrm{~h}$ with continuous drug re-addition (or DMSO alone in control cultures) for at least three 96 h-cycles. As shown in Figure-2, both inhibitors effectively reduced cell growth in primary cell culture, in nevirapine and efavirenz treated cells, comparing with control, with a stable effect during prolonged exposure. In Figure-2, we also show the relative rate of proliferation in nevirapine and efavirenz RCC primary culture relative to control culture (tumor derived primary RCC culture, not treated). During the first cycle, the inhibition relative to control remained at approximately $68 \%$ for nevirapine sample and $72 \%$ for efavirenz one (Figure-2). After the second and the third cycle, treated cells reduced the rate of their proliferation respectively to $31 \%$ (nevirapine treated) and $33 \%$ (efavirenz treated); it is clear that RT inhibitors were responsible for a $60 \%$ growth inhibition compared to control. We also defined the relative counts regarding cell death obtaining values similar to control (Figure-2).

\section{RT Inhibitors Induce Differentiation in RCC Cells}

It was relevant to determine whether RT inhibitors induced a differentiation-like state concomitant with reduced cell growth. We first examined RCC cells, which acquire a typical dendritic-like phenotype in response to certain inducers of differentiation (14). 
A
옴
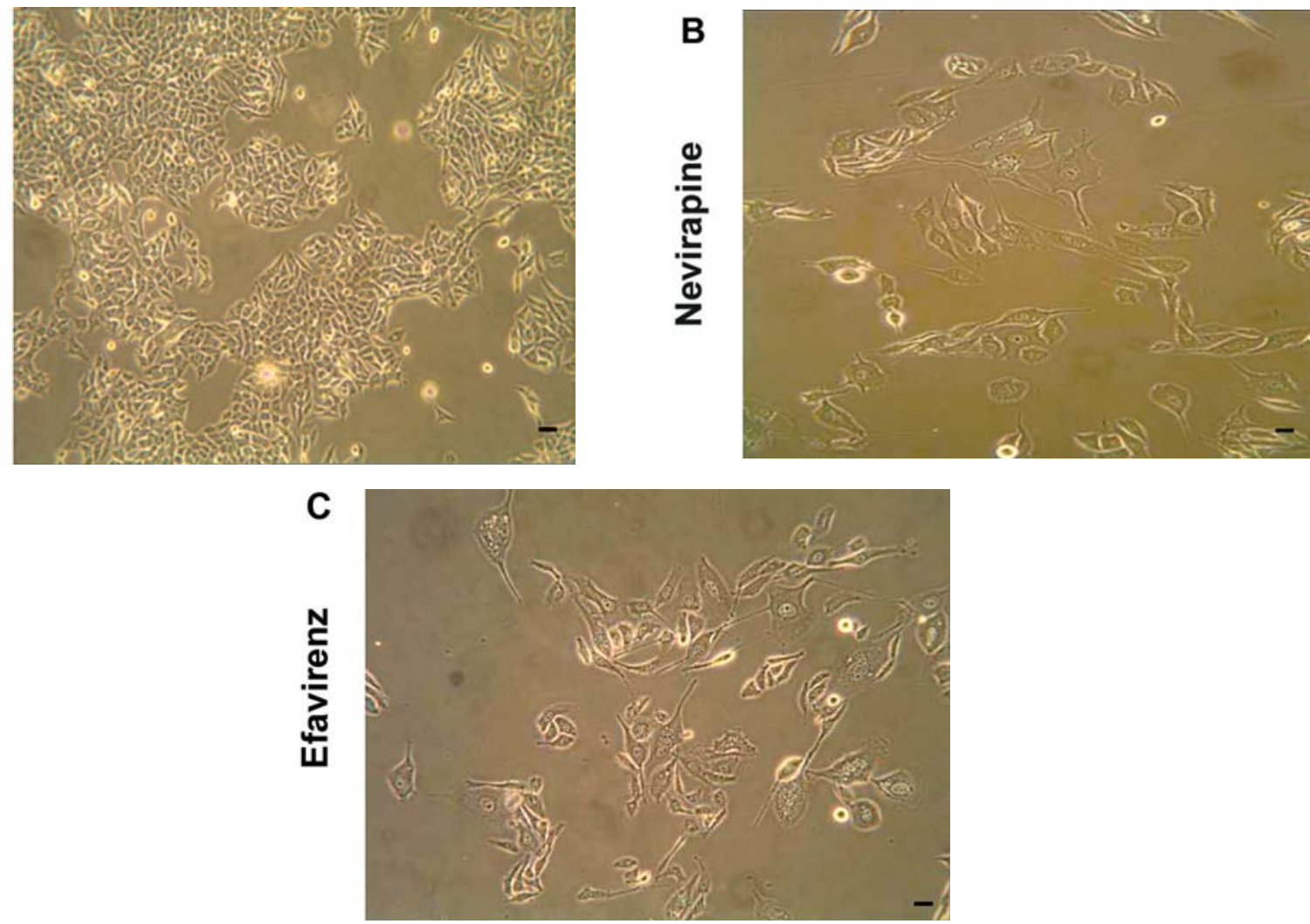

D

\section{Proliferation Rate}

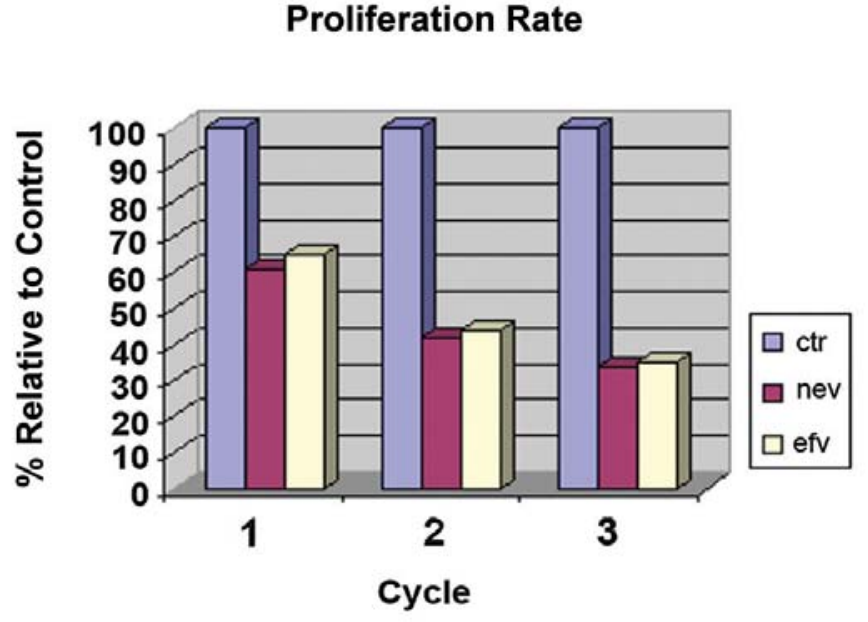

$\mathbf{E}$

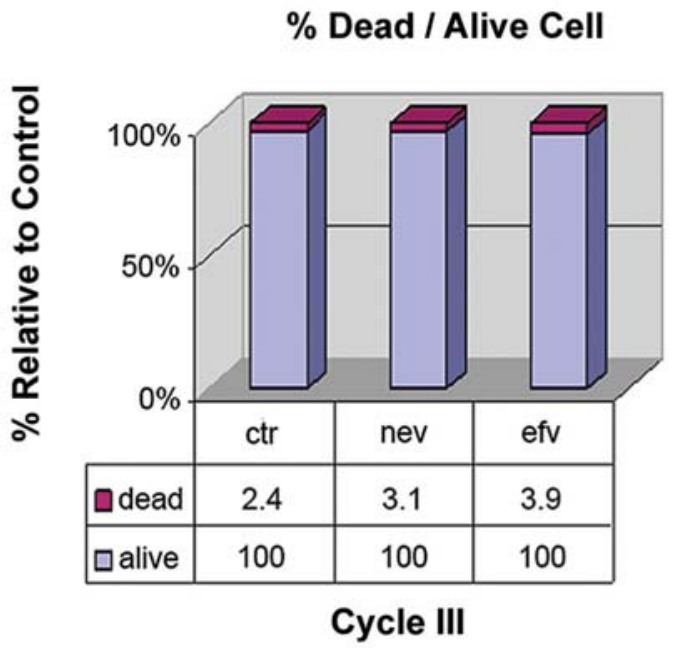

Figure 2-Renal carcinoma cells in vitro treatment with RT inhibitors. Treated RCC primary culture compared to ctr DMSO cultured cells (A), observed under phase-contrast microscope. Morphological differentiation in nevirapine (B) and efavirenz (C), bar, $10 \mu \mathrm{m}$. D) Inhibition of proliferation by anti - RT drugs: cell growth in cultures treated with DMSO (control, blue), nevirapine (nev, pink) and efavirenz (efv, white). Cells were counted and re-plated every 96 hor three cycles (1-3). Counted cells are expressed as the \% of controls, taken as 100. Values represent pooled data from two experiments. E) Relative percentage of dead treated and un-treated cells relative to total alive cell; counted cells are expressed as the \% total alive cell, taken as 100. Data represent medium values from three cycles. 

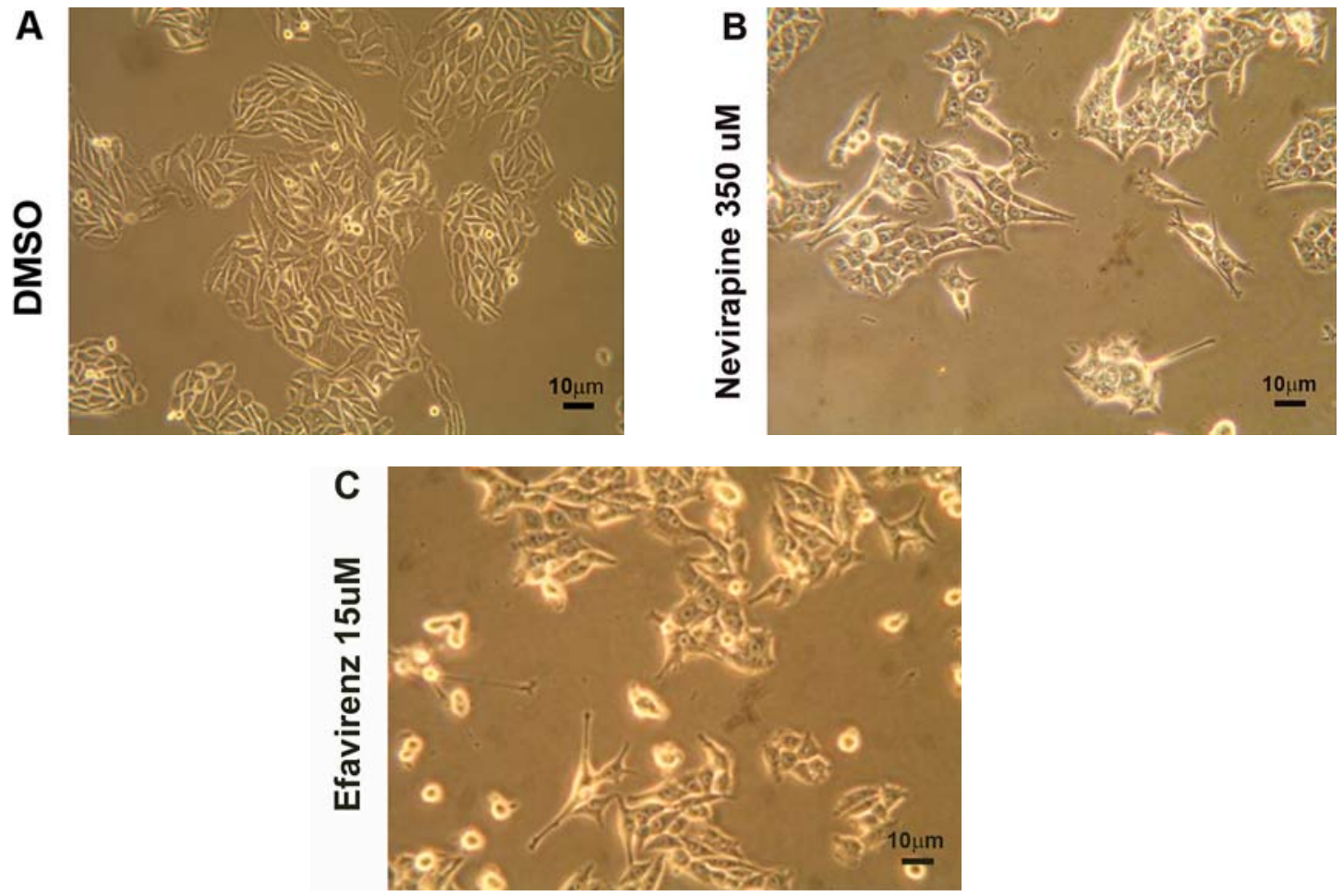

Figure 3 - Morphological differentiation of RCC in vitro primary culture after drug treatment: A) DMSO - RCC primary cell culture, control; B) In primary culture, nevirapine incubation determined dendritic like cell shape; C) As in nevirapine, also in efavirenz treated cells, there is a morphological differentiation: in this case, the dendritic extensions are less evident, but they are also present, indicating a general redistribution of cytoplasmic shape. Bars, $10 \mu \mathrm{m}$.

As shown in Figure-3, morphological change, revealed by cell shape and dendritic-like extensions, became evident within 4-5 days ( $96 \mathrm{~h}$ ) of exposure to nevirapine or efavirenz, compared to DMSO-treated controls.

\section{RT Enzymatic Activity Reduction in RCC Primary Cell Culture}

In a previous study we detected a functional RT activity in murine F9 cell line and in several human cell line (6). To assess whether an endogenous RT is also functional in RCC cell culture, we tested the ability of cell-free lysates prepared from in vitro RCC cell culture (tissue derived primary culture), inhibitors treated and not treated (DMSO), to retrotranscribe purified MS2 phage RNA. Lysate aliquots from RCC cell line (DMSO), nevirapine treated RCC cell line and efavirenz treated RCC cell line were incubated with purified MS2 phage RNA. Incubation mixtures where then subjected to direct PCR amplification using MS2-specific oligonucleotide pairs to establish whether MS2 cDNA molecules had been newly synthesized. As shown in Figure-4, a MS2-specific cDNA product of the expected size (112 bp) was retrotranscribed from the RNA template by RCC cell line lysates (lane 1 and 2). There was no difference between the RT activity in free RCC line cell culture and RCC cell culture in presence of $0.1 \%$ DMSO.

The 112 bp-long cDNA product was only obtained when cell lysates and phage RNA were incubated together, but not when RNA (lane 7) or lysate (lane 8) were omitted from incubation mixtures.

Furthermore, we sought to establish whether RT activity was sensitive to inhibition by nevirapine and efavirenz. The mechanism of nevirapine action is well characterized and the binding site of the molecule maps a hydrophobic pocket of the RT p66 subunit, 


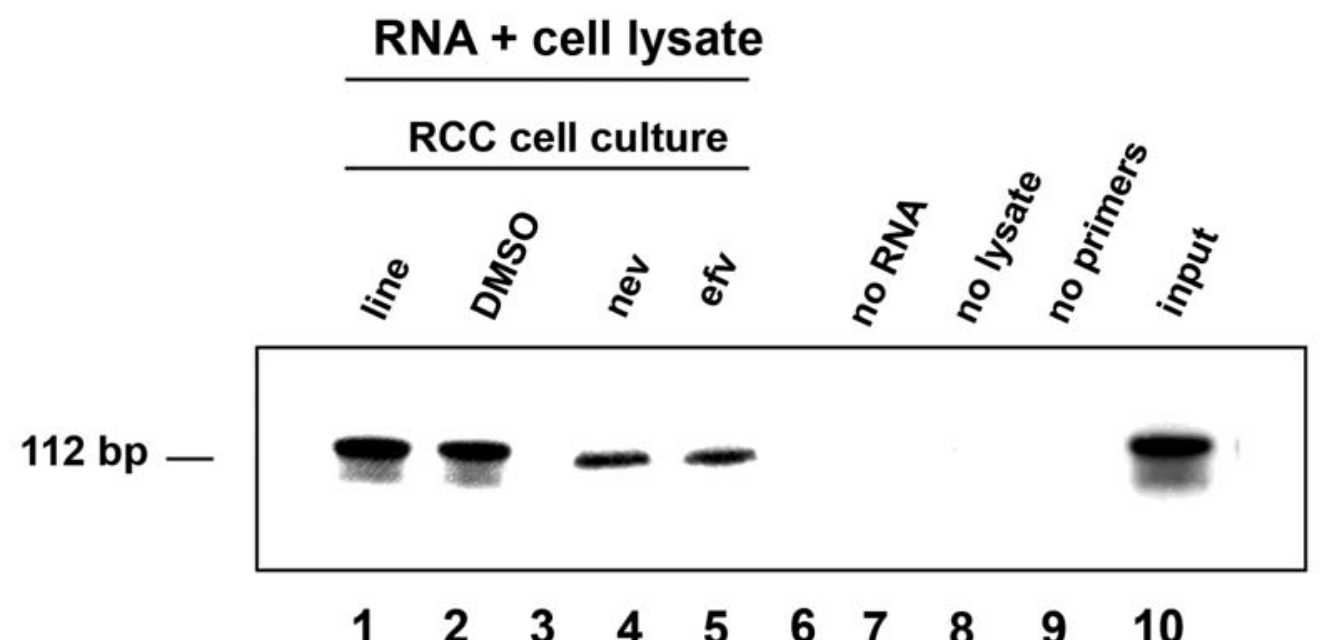

Figure 4 - Functional assay of RT activity from in vitro RCC cell culture lysates and inhibition by nevirapine and efavirenz. Endogenous RT activity was tested in vitro using MS2 phage RNA as the template and lysates from RCC primary culture cell line (lane 1) and DMSO in vitro RCC cell culture (lane 2, 4 and 5). Endogenous RT activity was tested in the absence (lane 1 and 2) or presence of RT inhibitors (nevirapine, lane 4; efavirenz, lane 5). Negative controls were selectively depleted of MS2 RNA (lane 7), cell lysate (lane 8) or PCR primers (lane 9). A positive control for the reaction utilized commercial RT instead of cell-lysate (lane 10).

near - though not overlapping with - the polymerase active site.

Preincubation of cell lysates with nevirapine and efavirenz inhibited retrotrascription of the RNA template significantly, though not totally, as shown in Figure-4, lanes 4 and 5, respectively. These results indicate that an endogenous RT activity is biologically functional in RCC in vitro cell culture and is sensitive to inhibition by nevirapine and efavirenz.

\section{COMMENTS}

This work highlights two unexpected aspects of the human genome that have implications for cancer: first, LINE L-1 elements, derived from active retroposon sequences, described by Brouha and coll. (15), have been identified as active components of a mechanism involved in the control of cell differentiation and proliferation; second, pharmacological inhibition of the endogenous RT activity which they encode, can restore control of these traits in transformed cells.
The RT inhibitors nevirapine and efavirenz, used in our study, share a common biochemical mechanism of action by binding the hydrophobic pocket in the p66 of retroviral reverse transcriptase (HIV) of RT enzymes. Although originally designed to target the HIV encoded RT, nevirapine was also able to inhibit the endogenous retro-transcriptase activity present in non infected cells (6) as shown in a highly sensitive in vitro assay (7). We also demonstrated that both drugs reduce proliferation of RCC primary cultures derived transformed cells, largely independent from cell death. Concomitant with this, RT inhibitors treatment induced a morphological transformation of the cells shape. The induction of morphological changes is rapid, different from phenotypic changes elicited by inhibitors of the telomerase-associated RT (TERT), which require an extensive treatment period which can be as long as 120 days (16).

Cytokine therapies are used in treatment of metastatic renal cell carcinoma.. However, these new biological agents only provide clinical benefit to a small subset of patients and are associated with significant toxicity (2-4). A better understanding of the 
molecular biology of RCC has identified the vascular endothelial growth factor and platelet-derived growth factor signaling pathways as rational targets for anticancer therapy. The multitargeted receptor tyrosine kinase inhibitors sunitinib and sorafenib have both demonstrated improved efficacy as second-line therapy in patients with RCC. Based on these partial results, the discovery of new antitumoral targets checked by antiretroviral agents could suggest the possibility of a synergic therapy against neoplastic proliferation. Therefore, nevirapine and efavirenz could produce a cytostatic effect permitting multikinases inhibitors to enhance their tumor regression activity.

Together with growth reduction and induction of partial differentiation, RT inhibition was responsible for the reprogramming of gene expression: this implicates endogenous RT in modulation of expression for genes that promote the transition from highly proliferating, transformed phenotypes to low proliferating, differentiated phenotypes, suggesting that genome function could be the ultimate target of pharmaceutical inhibition of RT activity.

In the present study, we analyzed the expression of four genes, indicated as molecular biomarkers in RCC cells: AFP, NNMT, CD70, FRA 2 and p21KIP1 genes.

CD70 gene is strongly down-regulated in treated RCC derived primary culture, as regards control of DMSO tumor cell culture, and this pattern is very similar to differentiated renal cells; although the role of this gene in cancer development and progression remains unclear, the encoded protein (type II trans-membrane glycoprotein) seems to mediate the interaction between $\mathrm{T}$ and B-lymphocytes and the natural killer cell-activation; it is also implicated in processes like cell proliferation, cell to cell signaling and induction of apoptosis by binding to its receptor CD27 (17). In several studies, high expression of CD70 has been found in malignant lymphomas and nasopharyngeal tumors (18) and in all examined clear cell tumors, whereas no expression occurred in the related normal epithelial cells.

Given that RCC can be considered as an immunogenic tumor, it is tempting to speculate that a CD70 over-expression can be associated to a possible immuno-escape for clear cell RCC: a strong reduction in expression level after RT inhibitors could induce a reprogramming status where cell progression is arrested and the cell partially differentiated.

The Fos protein FRA2 forms transcription factor complexes and has been described as a regulator of cell proliferation, differentiation and transformation and in some cases the expression of FOS gene has also been associated with apoptotic cell death (19). The mRNA expression pattern of FRA2 is differently regulated in treated and untreated cell culture: in the first case, the gene was up-regulated, while in the control sample the expression level was much lower.

AFP gene encodes alpha-fetoprotein, a major plasma produced by the yolk sac and liver during fetal life. Alpha-fetoprotein expression in adults is often associated with hepatoma or teratoma. AFP mRNA is a more reliable marker of metastasis compared to serum AFP (20).

p 27 KIP1 gene encodes a cyclin-dependent kinase inhibitor, which shares a limited similarity with CDK inhibitor CDKN1A/p21. The encodec protein binds to and prevents the activation of cyclin E-CDK2 or cyclin D-CDK4 complexes, and thus controls the cell cycle progression at G1. The degradation of this protein, which is triggered by its CDK dependent phosphorylation and subsequent ubiquitination by SCF complexes, is required for the cellular transition from quiescence to the proliferative state.

Decreased p27 expression has been shown to be associated with aggressive tumor behavior and decreased patient survival in numerous human malignancies (12). Expression level of p27 mRNA in treated renal carcinoma cells is strongly up-regulated demonstrating that cells could reverse to a quiescent condition, prior differentiation.

Changes in gene expression are not inherited through cell division, but are reversible when RT inhibition is released (data not shown). The reversibility of examined features after release of the inhibition suggest that LINE-1 encoded RT is part of an epigenetic mechanism that can modulate gene expression and contributes to the molecular mechanisms underlying cell proliferation and differentiation.

\section{CONCLUSIONS}

The prospect of using RT inhibitors in RCC cancer therapy could have obvious advantages given 
their resistance to many therapeutical approaches such as chemo-, radio- and immunotherapy.

The finding of a stable inhibition of endogenous reverse transcriptase activity in tumor or proliferative RCC cells opens the possibility for the involvement of retro-elements and retrotrasposon-sequences in the control of the proliferative process. In this study, we attempted to establish a new therapeutic approach to arrest in vitro cell growth in a RCC-derived primary cell culture as a possible useful application in cancer treatment.

\section{ACKNOWLEDGEMENT}

This work was supported by National Italian Institute of Health.

\section{CONFLICT OF INTEREST}

None declared.

\section{REFERENCES}

1. Thoenes W, Störkel S, Rumpelt HJ, Jacobi GH: Renal cell carcinoma- a classification based on cytomorphological criteria. Zentralbl Allg Pathol. 1986; 132: 503-13.

2. Motzer RJ, Hutson TE, Tomczak P, Michaelson MD, Bukowski RM, Rixe O, et al.: Sunitinib versus interferon alfa in metastatic renal-cell carcinoma. N Engl J Med. 2007; 356: 115-24.

3. Escudier B, Eisen T, Stadler WM, Szczylik C, Oudard S, Siebels M, et al.: Sorafenib in advanced clear-cell renal-cell carcinoma. N Engl J Med. 2007; 356: 12534. Erratum in: N Engl J Med. 2007; 357: 203.

4. Hudes G, Carducci M, Tomczak P, Dutcher J, Figlin R, Kapoor A, et al.: Temsirolimus, interferon alfa, or both for advanced renal-cell carcinoma. N Engl J Med. 2007; 356: 2271-81.

5. Costa LJ, Drabkin HA: Renal cell carcinoma: new developments in molecular biology and potential for targeted therapies. Oncologist. 2007; 12: 1404-15.

6. Mangiacasale R, Pittoggi C, Sciamanna I, Careddu A, Mattei E, Lorenzini R, et al.: Exposure of normal and transformed cells to nevirapine, a reverse transcriptase inhibitor, reduces cell growth and promotes differentiation. Oncogene. 2003; 22: 2750-61.

7. Pyra H, Böni J, Schüpbach J: Ultrasensitive retrovirus detection by a reverse transcriptase assay based on product enhancement. Proc Natl Acad Sci U S A. 1994; 91: 1544-8.

8. Diegmann J, Junker K, Gerstmayer B, Bosio A, Hindermann W, Rosenhahn J, et al.: Identification of CD70 as a diagnostic biomarker for clear cell renal cell carcinoma by gene expression profiling, real-time RT-PCR and immunohistochemistry. Eur J Cancer. 2005; 41: 1794-801.

9. Shirsat NV, Shaikh SA: Overexpression of the immediate early gene fra- 1 inhibits proliferation, induces apoptosis, and reduces tumourigenicity of c6 glioma cells. Exp Cell Res. 2003; 291: 91-100.

10. Giannelli G, Marinosci F, Trerotoli P, Volpe A, Quaranta M, Dentico P, et al.: SCCA antigen combined with alpha-fetoprotein as serologic markers of HCC. Int J Cancer. 2005; 117: 506-9.

11. Imura $\mathrm{A}$, Hori $\mathrm{T}$, Imada $\mathrm{K}$, Kawamata S, Tanaka $\mathrm{Y}$, Imamura S, et al.: OX40 expressed on fresh leukemic cells from adult T-cell leukemia patients mediates cell adhesion to vascular endothelial cells: implication for the possible involvement of OX40 in leukemic cell infiltration. Blood. 1997; 89: 2951-8.

12. Anastasiadis AG, Calvo-Sanchez D, Franke KH, Ebert T, Heydthausen M, Schulz WA, et al.: p27KIP1expression in human renal cell cancers: implications for clinical outcome. Anticancer Res. 2003; 23: 21721.

13. Sciamanna I, Landriscina M, Pittoggi C, Quirino M, Mearelli C, Beraldi R, et al.: Inhibition of endogenous reverse transcriptase antagonizes human tumor growth. Oncogene. 2005; 24: 3923-31.

14. Sauane M, Gopalkrishnan RV, Sarkar D, Su ZZ, Lebedeva IV, Dent P, et al.: MDA-7/IL-24: novel cancer growth suppressing and apoptosis inducing cytokine. Cytokine Growth Factor Rev. 2003; 14: 35-51.

15. Brouha B, Schustak J, Badge RM, Lutz-Prigge S, Farley AH, Moran JV, et al.: Hot L1s account for the bulk of retrotransposition in the human population. Proc Natl Acad Sci U S A. 2003; 100: 5280-5.

16. Damm K, Hemmann U, Garin-Chesa P, Hauel N, Kauffmann I, Priepke H, et al.: A highly selective telomerase inhibitor limiting human cancer cell proliferation. EMBO J. 2001; 20: 6958-68.

17. Wischhusen J, Jung G, Radovanovic I, Beier C, Steinbach JP, Rimner A, et al.: Identification of CD70-mediated apoptosis of immune effector cells as a novel im- 
mune escape pathway of human glioblastoma. Cancer Res. 2002; 62: 2592-9.

18. Douin-Echinard V, Bornes S, Rochaix P, Tilkin AF, Peron JM, Bonnet J, et al:: The expression of CD70 and CD80 by gene-modified tumor cells induces an antitumor response depending on the MHC status. Cancer Gene Ther. 2000; 7 : 1543-56.

19. Tilghman SM: The structure and regulation of the alpha-fetoprotein and albumin genes. Oxf Surv Eukaryot Genes. 1985; 2: 160-206.

\section{Correspondence address:}

Dr. Carmine Pittoggi

S.B.G.S.A, Italian National Institute of Health

Viale Regina Elena, 299

Rome, 00161, Italy

E-mail: carminepittoggi@hotmail.com
20. Yao F, Guo JM, Xu CF, Lou YL, Xiao BX, Zhou WH, et al.: Detecting AFP mRNA in peripheral blood of the patients with hepatocellular carcinoma, liver cirrhosis and hepatitis. Clin Chim Acta. 2005; 361: 119-27.
Accepted after revision: May 23, 2008 